COMMENT. Partial cerebral folate deficiency may occur as a secondary abnormality in acquired CNS disorders and in genetic and congenital encephalopathies. Severe deficiencies are caused by inborn errors of folate metabolism or cerebral transport defects across the blood-CSF barrier (FOLR1 mutation). In the above study, children younger than 1 year had epilepsy (infantile spasms and neonatal seizures), perinatal asphxia or CNS infection. Older children were diagnosed with inherited disease, mitochondrial disorders or cerebellar atrophy syndrome. The mechanism of acquired cerebral folate deficiency in neurologic disorders is not definitely determined. Children with seizure disorders are at risk of low serum folate, and antiepileptic drugs may be a contributing factor in some cases. The value of folinic acid supplement in cerebral folate deficiency is under investigation.

\title{
NEUROLOGIC FEATURES OF WOLFRAM SYNDROME
}

Researchers at Nice, Marseille, and Montpellier-Nimes, France, studied the nature and frequency of neurologic manifestations in 59 patients with Wolfram syndrome with genotype-phenotype correlations. Wolfram syndrome is a genetically heterogeneous disease characterized by juvenile onset diabetes mellitus and optic atrophy, with progressive development of diabetes insipidus, sensorineural deafness, renal, and neuropsychiatric disorders. In this series of patients, median age at diagnosis was 10 years (range, $4-43$ years). The onset of neurologic symptoms in 31 (53\%) patients was at a median age of 15 years. These involved dysfunction of brain stem or cerebellum in $17 / 31(55 \%)$ and included cerebellar ataxia (45\%), frequently associated with dysarthria, dysphagia, or nystagmus. Peripheral neuropathy occurred in $39 \%$ cases, and cognitive impairment in $32 \%$. Eight patients presented with epilepsy, mainly generalized. MRI was abnormal in 13 patients, showing cerebral, cerebellar and brainstem atrophy. WFS1 mutations (10/56 novel) were identified in $90 \%$ of patients. The age of onset of neurologic symptoms was not correlated with genotype, and homozygosity or compound heterozygosity for missense mutation did not influence their development. The location of the WFS1 mutations may play a role in the development of neurologic manifestations. (Chaussenot A, Bannwarth S, Rouzier C et al. Neurologic features and genotypephenotype correlation in Wolfram syndrome. Ann Neurol March 2011;69:501-508). (Respond: $\operatorname{Pr}$ Veronique Paquis-Flucklinger, Nice, France. E-mail: paquis( $a$ hermes.unice.fr).

COMMENT. The prevalence of Wolfram syndrome in N America is estimated at $1 / 100,000$, and neurologic manifestations have previously received limited attention, especially in children. The authors emphasize the importance of diagnosis and follow-up of neurologic complications because of fatalities associated with brainstem involvement. Genetic testing is recommended to optimize family counseling.

Neurologic findings in 5 patients with Wolfram syndrome, also known as DIDMOAD syndrome, included dysarthria, seizures, anosmia, nystagmus, ataxia, and changes in the EEG, electroretinogram and evoked potentials. Four patients had abnormal CT scans with atrophy of the brainstem and cerebellum, similar to olivopontocerebellar atrophy (OPCA). Wolfram syndrome includes phenotypic manifestations of OPCA. (Leiva-Santana C et al. Rev Neurol (Paris) 1993;149(1):26-29). 